\title{
Adaptação de lentes de contato após cirurgia refrativa
}

\author{
Contact lens fitting after refractive surgery
}

\author{
Adimara da Candelária Renesto ${ }^{1}$ \\ César Lipener ${ }^{2}$
}

Trabalho realizado no Setor de Lente de Contato e Refração do Departamento de Oftalmologia da Universidade Federal de São Paulo - UNIFESP.

${ }^{1}$ Estagiária do Setor de Lente de Contato e Refração do Departamento de Oftalmologia da Universidade Federal de São Paulo - UNIFESP.

${ }^{2}$ Chefe do Setor de Lente de Contato e Refração do Departamento de Oftalmologia e Mestre em Oftalmologia pela Universidade Federal de São Paulo - UNIFESP.

Endereço para correspondência: Rua Carlos Sampaio, 94/22 - São Paulo (SP) CEP 01333-020

E-mail: adimararenesto@uol.com.br

Recebido para publicação em 09.03.2004

Versão revisada recebida em 04.10.2004

Aprovação em 30.10.2004

Nota Editorial: Pela análise deste trabalho e por sua anuência na divulgação desta nota, agradecemos ao Dr. Adamo Lui Netto.

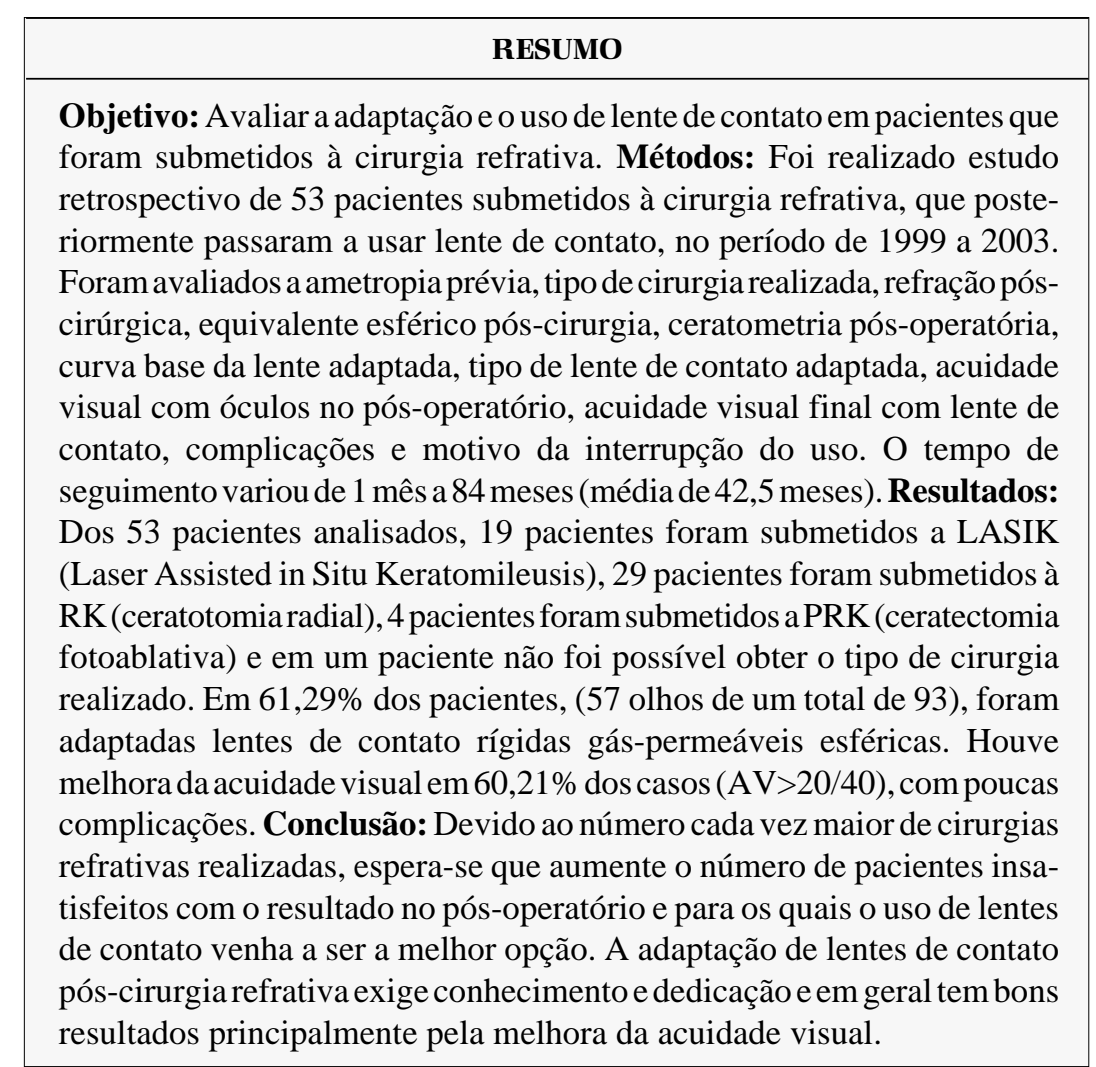

Descritores: Lentes de contato; Erros de refração/cirurgia; Acuidade visual; Estudos retrospectivos

\section{INTRODUÇÃOO}

Atualmente, a cirurgia refrativa tem sido grande destaque não só no meio oftalmológico como em toda a sociedade. Estima-se que mais de 1 milhão de procedimentos cirúrgicos refrativos foram realizados somente nos Estados Unidos no ano de $2000^{(1)}$. As cirurgias mais realizadas foram: PRK e LASIK.

Face ao grande número de cirurgias e ocorrência de hipocorreções, hipercorreções e astigmatismo, os pacientes podem necessitar novamente de correção visual, como óculos ou lente de contato. Além das complicações acima citadas, têm-se também córneas multifocais e flutuação da visão ${ }^{(2-4)}$. Em estudo feito por Astin e col. ${ }^{(5-6)}$, as lentes de contato levaram a uma melhora da acuidade visual, e em casos de moderado astigmatismo irregular houve significante melhora em relação ao uso de óculos. Nenhuma reação adversa ao uso das lentes de contato foi encontrada. Também em outro estudo(7) $^{(7)}$ mostram que o uso das lentes de contato é em algumas 
vezes a única alternativa para pacientes com astigmatismo irregular após cirurgia refrativa, levando à melhora da acuidade visual e boa tolerância.

No presente, o objetivo foi analisar o processo de adaptação de lente de contato em pacientes que foram submetidos à cirurgia refrativa, considerando o tipo de lente de contato adaptada, medidas ceratométricas, padrão fluoresceínico da lente de contato à lâmpada de fenda, acuidade visual final do paciente, tempo de seguimento, complicações e motivo da interrupção ao uso das lentes de contato.

\section{MÉTODO}

Foi realizado estudo retrospectivo com 53 pacientes que foram submetidos à cirurgia refrativa, e que procuraram o serviço para adaptar lentes de contato entre dezembro de 1999 a junho de 2003 no Setor de Lentes de Contato da Universidade Federal de São Paulo (UNIFESP). Todos os pacientes avaliados não apresentavam outras doenças oculares associadas. Critérios de exclusão: ceratocone e catarata. Os pacientes foram encaminhados para adaptação com lente de contato devido a complicações decorrentes da cirurgia refrativa. Os seguintes parâmetros foram avaliados na adaptação da lente de contato: idade; sexo; data da cirurgia refrativa; ametropia prévia (obtida através do prontuário médico); tipo de cirurgia realizada (também obtida através do prontuário médico); data de início da adaptação da lente de contato; refração e equivalente esférico presente; ceratometria $(\mathrm{K})$ pós-operatória; curva base da lente de contato adaptada; tipo de lente de contato adaptada; acuidade visual com óculos no pós-operatório; acuidade visual final com lente de contato; tempo de seguimento; complicações; motivo da interrupção do uso da lente de contato. A lente de prova inicialmente usada para adaptação foi lente de contato rígida gás-permeável esférica (LCRGPE), porém, em alguns pacientes foi necessário usar outros tipos de lentes de contato.

No processo de adaptação das LCRGPE, usamos lentes de diâmetro grande, pois ajuda a obter boa centralização da LC. Em relação à ceratometria, utilizamos de preferência a medida mais plana da córnea $(\mathrm{K})$ do pré-operatório (quando não é possível, usamos a medida do pós-operatório). Em alguns casos, a curva base da LC também pode ser baseada na topografia corneana pós-operatória.

Foram analisados a centralização, mobilidade, diâmetro, tolerância e o padrão da lente de contato após instilação de fluoresceína, sendo estes parâmetros observados no exame à lâmpada de fenda.

\section{RESULTADOS}

Foram avaliados 53 pacientes submetidos à cirurgia refrativa, e que posteriormente necessitaram de adaptação de lente de contato. Destes, 31 pacientes eram do sexo feminino $(58,49 \%)$ e 22 pacientes eram do sexo masculino $(41,50 \%)$. A idade variou de 21 a 70 anos, com média de 45,5 anos. Em relação aos outros parâmetros analisados, observamos o seguinte:
Data da cirurgia refrativa: os pacientes avaliados foram submetidos à cirurgia refrativa dentro de um período de 25 anos a partir de 1979; ametropia prévia: 13 pacientes apresentavam miopia $(24,52 \%)$; 6 pacientes apresentavam astigmatismo miópico composto $(11,32 \%)$; 1 paciente apresentava astigmatismo hipermetrópico composto $(1,88 \%)$; em 33 pacientes não foi possível obter este dado, pois não havia no prontuário; tipo de cirurgia refrativa: em 19 pacientes foi realizado LASIK $(35,84 \%)$; em 29 pacientes foi realizado RK (54,71\%); em 4 pacientes foi realizado PRK (7,54\%); em 1 paciente não foi possível saber o tipo de cirurgia realizada, pois este dado não constava no prontuário; refração pós-cirurgia: o componente esférico variou de -23,00DE a +8,50 DE; o componente cilíndrico variou de -6,00 DC a -0,25 DC; ceratometria (K) pós-operatório e curva base da lente de contato adaptada: estes dados encontram-se relacionados nos quadros 1 e 2; tipo de lente de contato adaptada: em 57 olhos foram adaptados LCRGPE (61,29\%); em 12 olhos foram adaptados LCRGP multiesférica de diâmetro fixo (12,90\%); em 7 olhos foram adaptados LC gelatinosa convencional (7,52\%); em 4 olhos foram adaptados LC gelatinosa tórica $(4,30 \%)$; em 5 olhos foram adaptados lentes de contato de geometria reversa (5,37\%); em 2 olhos foram adaptados LC Sopper (2,15\%); em 2 olhos (mesmo paciente), foram adaptados LC esclerais $(2,15 \%)$; em 2 olhos (mesmo paciente), foram adaptados LCRGP híbridas (2,15\%); em 2 olhos (mesmo paciente), foram adaptados LC gelatinosa bifocal $(2,15 \%)$; equivalente esférico pós-cirurgia: variou de -25,50 DE a +6,50 DE, encontrando-se cada medida relacionada no quadro ; tempo de seguimento: variou de 1 mês a 84 meses, com média de 42,5 meses; acuidade visual final: de 93 olhos adaptados com LC, $60,21 \%$ apresentaram AV melhor do que 20/40. Dentro destes 60,21\%, 43 olhos (46,23\%) apresentaram acuidade visual maior do que 20/40, e 13 olhos $(13,97 \%)$ tiveram acuidade visual maior do que 20/25; nos quadros 1 e 2, observamos a acuidade visual após a cirurgia com óculos e com LC, havendo melhora quando em uso da lente de contato; complicações: $45(84,90 \%)$ dos pacientes não apresentaram complicações durante o período de seguimento; nos outros 8 pacientes, $(15,09 \%)$, ocorreram: 4 pacientes apresentaram ceratite no processo de adaptação das lentes de contato; 2 pacientes apresentaram desepitelização corneana durante os testes; 1 paciente apresentou irritação e vermelhidão ocular após a adaptação e 1 paciente não sentiu conforto com o uso das LC; motivo da interrupção do uso das LC: 2 pacientes não apresentaram melhora da acuidade visual com o uso das LC; em 2 pacientes não se verificou a melhora da ceratite; 1 paciente não ficou satisfeito com o uso das LC; 1 paciente apresentou diplopia durante o uso das LC e em 1 paciente não houve melhora da desepitelização corneana.

\section{DISCUSSÃO}

Com o crescente aumento do número de cirurgias refrativas realizadas atualmente, é esperado que haja um certo número de complicações pós-operatórias. Como consequiência, alguns pacientes necessitam novamente usar correção óptica, como ócu- 
los ou lente de contato. As complicações mais freqüentes são hipercorreção, hipocorreção, astigmatismo irregular e córneas multifocais. Nestes casos, muitos pacientes necessitam do uso de lente de contato para melhorar a acuidade visual.

Em alguns estudos ${ }^{(7)}, o$ astigmatismo irregularé um dos mais difíceis e frustrantes problemas após cirurgia refrativa, e algumas vezes a única alternativa para esses pacientes é o uso de LC. Outros trabalhos ${ }^{(1)}$ enfatizaram que as LC geralmente melhoram a distorção visual causada pela irregularidade corneana.

O processo de adaptação de lente de contato em pacientes submetidos à cirurgia refrativa requer mais cuidados, mais tempo e apresenta maior grau de dificuldade do que em pacientes não operados. Além disso, temos que levar em consideração o fato de que esses pacientes são mais refratários em usar novamente lentes de contato, pois a expectativa destes em relação à cirurgia era diminuir a necessidade de correção óptica.

Em um estudo caso-controle ${ }^{(1)}$ envolvendo 30 pacientes operados de cirurgia refrativa e 30 pacientes não operados, verificou-se que olhos submetidos à cirurgia refrativa utilizaram mais lentes de prova do que o grupo controle. A acuidade visual com as LC foi estatisticamente pior no grupo operado em relação ao grupo controle. Assim, observaram que córneas alteradas pela cirurgia refrativa (incisional e lamelar) são mais difíceis de adaptar LC que pacientes não operados; verificaram também que as LCRGP (lentes de contato rígidas gáspermeáveis) geralmente melhoram a distorção visual causada pela irregularidade corneana.

Em nosso serviço iniciamos a adaptação da LC com LCRGPE (lente de contato rígida gás-permeável esférica), independente do tipo de cirurgia refrativa realizada, LASIK, PRK ou RK, e para cada tipo de cirurgia existem certas particularidades no processo de adaptação. Tanto no LASIK como no PRK $^{(5,8-9)}$ a periferia e média periferia corneana são menos afetadas. Já na $\mathrm{RK}^{(10-11)}$, a curvatura corneana é totalmente afetada, com aplanamento da área central e encurvamento da meia periferia (cotovelo), ocasionando maior dificuldade na adaptação da lente de contato ${ }^{(12)}$. Independentemente também do tipo de cirurgia, todas podem levar à hipercorreção, hipocorreção, descentração da zona óptica, astigmatismo irregular e flutuação da visão.

$\mathrm{Na}$ adaptação das LCRGPE utilizamos lentes de diâmetro grande, $\mathrm{K}$ pré-operatório ou pós-operatório e verificamos o padrão da LC à lâmpada de fenda. Em alguns casos, a curva base da LC também pode ser baseada na topografia corneana pós-operatória ${ }^{(12)}$.

Espera-se uma LC com boa centralização, bom padrão fluoresceínico, boa mobilidade e conforto para o paciente. Nos casos insatisfatórios utilizamos outros tipos de lente de contato, como as LCG (lente de contato gelatinosa convencional) ${ }^{(13)}$ LCG (tórica), LCG (bifocal), LC multiesférica de diâmetro fixo, LC Sopper, LCGR (geometria reversa) ${ }^{(14)}$, LC escleral e LCRGP híbrida, visando sempre melhorar a acuidade visual e ou conforto do paciente. Em nosso estudo, verificamos uma melhora da acuidade visual com o uso das LC em relação ao uso dos óculos após a cirurgia refrativa.
Em um estudo realizado ${ }^{(2)}, 32$ olhos de 28 pacientes que foram submetidos à $\mathrm{RK}$ apresentaram hipercorreção após a cirurgia, e foram posteriormente adaptados com LC. Em 23 casos foram adaptadas LCRGP, e em 9 casos LCG. Oito olhos adaptados com LCG foram readaptados com LCRGP devido à visão inadequada ou desenvolvimento de neovascularização e 6 olhos usando LCRGP foram readaptados com LCG devido a problemas de conforto.

Assim, embora muitos pacientes prefiram o uso das LCG a as LCRGP, devemos levar em consideração as complicações decorrentes do uso de uma LCG após ceratotomia radial. Nos casos de ametropias esféricas, as lentes de siloxane-hidrogel podem ser consideradas ${ }^{(2)}$.

Em alguns trabalhos ${ }^{(11)}$, a topografia corneana de pacientes após RK demonstra um grande aplanamento central, sendo as LCRGP as mais adequadas para adaptação após RK; em outros estudos ${ }^{(9-13)}$, os pacientes submetidos tanto a PRK como RK, foram adaptados com LCRGP e também com LCG, pois não toleraram as LCRGP, e também tiveram boa acuidade visual e maior conforto. Em um determinado estudo ${ }^{(13)}$ avaliou-se 29 pacientes, onde 18 pacientes foram adaptados com LCRGP e 11 pacientes foram adaptados com LCG. Nesse estudo, mais pacientes tiveram sucesso com as LCG comparados à LCRGP; as LCG também tiveram melhor centralização do que as rígidas, entretanto, as LCRGP ofereceram melhor visão para pacientes com astigmatismo irregular.

Atualmente, vários tipos de lente de contato e novos materiais tem sido lançados no mercado contribuindo para melhorar as adaptações. Um exemplo são as LC de geometria reversa $^{(12)}$ que possuem uma área central mais plana e uma curva reversa na periferia, visando se adequar ao novo formato da córnea. Outra vantagem dessas lentes é possuírem um alto DK, proporcionando mais segurança para o paciente.

Em nosso estudo, dos 93 olhos adaptados com LC, em 57 olhos $(61,29 \%)$ foram adaptados LCRGPE. Essas lentes possuíam um diâmetro grande levando a uma boa centralização, boa troca do filme lacrimal e boa acuidade visual.

Em um estudo prospectivo ${ }^{(7)}$ foram adaptadas diferentes tipos de LC (RGP, híbrida, tórica gel) em 29 olhos de pacientes que apresentavam astigmatismo irregular após cirurgia refrativa. O sucesso na adaptação foi obtido em 23 olhos $(79,31 \%)$, onde 14 olhos $(60,86 \%)$ foram adaptados com LCRGP; 6 olhos (26,08\%) com LCG; 3 olhos com LC híbridas. Os resultados mostraram que o uso das LC é uma boa, e algumas vezes a única alternativa para pacientes com astigmatismo irregular, sendo que as LCRGP levaram a uma melhor acuidade visual.

É importante no processo de adaptação das LC nesses pacientes utilizarmos todos os meios disponíveis para obtermos uma adaptação satisfatória, pois muitas vezes são pacientes que não estão motivados ao uso de LC. Devemos mostrar e ressaltar a esses pacientes a melhora da visão e o conforto com o uso das LC, além de acompanhá-los bem de perto e tentar mostrar-lhes que o uso das LC proporciona melhora da acuidade visual contribuindo para uma melhor qualidade de vida. 


\section{CONCLUSÃO}

Concluímos que a adaptação de lentes de contato após cirurgia refrativa tem trazido bons resultados àqueles pacientes que tiveram complicações pós-operatórias, principalmente com relação à melhora da acuidade visual. Apesar de terem sido usados vários tipos e materiais de lentes, as rígidas gáspermeáveis esféricas foram as que levaram a um melhor resultado na adaptação, principalmente em relação à grande melhora da acuidade visual.

\section{ABSTRACT}

Purpose: To evaluate the fitting and use of contact lens in patients submitted to refractive surgery. Methods: This was a retrospective study in 53 patients submitted to refractive surgery who later started to use contact lens, from 1999 to 2003. The parameters were: previous ametropia, refractive surgery procedure, ametropia after surgery, postoperative spherical equivalent, postoperative keratometry, base curve of fitted contact lens, contact lens design, final visual acuity with spectacles after surgery, final visual acuity with contact lens, complications and the reason for stopping the use. The follow-up of patients ranged from 1 month to 84 months (average of 42,5 months). Results: Of 53 evaluated patients, 19 patients had undergone LASIK (Laser Assisted in Situ Keratomileusis), 29 patients had undergone RK (radial keratotomy), 4 patients had undergone PRK (photorefractive keratectomy) and in one patient it was not possible to know which surgery was performed. $61.29 \%$ of the patients (57 eyes of a total of 93 eyes) were fitted with rigid gas-permeable lenses. There was an improvement of visual acuity in $60.21 \%$ of the cases (VA>20/40), with few complications. Conclusion: Due to the great number of performed refractive surgery, an increase in the number of patients unhappy with the postoperative result is expected, and for these patients, many times the use of contact lens is the best option. The fitting of contact lenses after refractive surgery demands knowledge, dedication and has good results principally regarding better visual acuity.

Keywords: Contact lenses; Refractive errors/surgery; Visual acuity; Retrospective studies

\section{REFERÊNCIAS}

1. Yeung KK, Olson MD, Weissman BA. Complexity of contact lens fitting after refractive surgery. Am J Ophthalmol. 2002;133(5):607-12.

2. Shivitz IA, Arrowsmith PN, Russel BM. Contact lenses in the treatment of patients with overcorrected radial keratotomy. Ophthalmology. 1987;94(8): 899-903.

3. Eggink FA, Beekhuis WH, Nuijts RM. Rigid gas-permeable contact lens fitting in LASIK patients for the correction of multifocal corneas. Graefes Arch Clin Exp Ophthalmol. 2001;239(5):361-6.

4. Rowsey JJ, Rubin ML. Refraction problems after refractive surgery. Surv Ophthalmol. 1988;32(6):414-20.

5. Astin CL, Gartry DS, McG Steele AD. Contact lens fitting after photorefractive keratectomy. Br J Ophthalmol. 1996;80(7):597-603.

6. Davis LJ. Rigid gas permeable extended wear (RGPEW) for the postoperative patient: a review and clinical observations. J Am Optom Assoc. 1994;65(3): 179-86. Review.

7. Alio JL, Belda JI, Artola A, Garcia-Lledó M, Osman A. Contact lens fitting to correct irregular astigmatism after corneal refractive surgery. J Cataract Refract Surg. 2002;28(10):1750-7.

8. Eggink FA, Beekhuis WH. Contact lens fitting in a patient with keratectasia after laser in situ keratomileusis. J Cataract Refract Surg. 2001;27(7):1119-23.

9. Astin CL. Contact lens fitting after photorefractive keratectomy: a comparison of two groups of patients. Ophthalmic Physiol Opt. 1995;15(5):371-4.

10. Bourque LB, Lynn MJ, Waring GO 3rd, Cartwright C. Spectacle and contact lens wearing six years after radial keratectomy in the prospective evaluation of radial keratectomy study. Ophthalmology. 1994;101(3):421-31.

11. McDonnell PJ, Garbus JJ, Caroline P, Yoshinaga PD. Computerized analysis of corneal topography as an aid in fitting contact lenses after radial keratotomy. Ophthalmic Surg. 1992;23(1):55-9.

12. Hersh PS, Ratnakaram R, Hersh D, Fry K. Diagnostic use of a rigid contact lens to show corneal topography abnormalities after laser refractive surgery. $\mathrm{J}$ Cataract Refract Surg. 2002;28(11):2054-7.

13. Lim L, Siow KL, Chong JS, Tan DT. Contact lens wear after photorefractive keratectomy: comparison between rigid gas permeable and soft contact lenses. CLAO J. 1999;25(4):222-7.

14. Lim L, Siow KL, Sakamoto R, Chong JS, Tan DT. Reverse geometry contact lens wear after photorefractive keratectomy, radial keratectomy, or penetrating keratoplasty. Cornea. 2000;19(3):320-4.

\section{Nos artigos enviados para publicação, o nome dos autores \\ e suas afiliações devem estar completos. Isso facilitará a indexação e os links com as bases de dados e o CV Lates.}

